\title{
Front Matter: Volume 11179
}

, "Front Matter: Volume 11179," Proc. SPIE 11179, Eleventh International Conference on Digital Image Processing (ICDIP 2019), 1117901 (14 August 2019); doi: $10.1117 / 12.2545164$

SPIE Event: Eleventh International Conference on Digital Image Processing (ICDIP SPIE. 2019), 2019, Guangzhou, China 


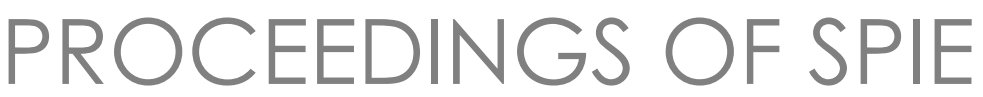

\section{Eleventh International Conference on Digital Image Processing (ICDIP 2019)}

Jenq-Neng Hwang

Xudong Jiang

Editors

10-13 May, 2019

Guangzhou, China

\section{Cosponsored by}

East China Normal University (China)

International Association of Computer Science and Information Technology (Singapore)

Hosted by

Sun Yat-Sen University (China)

Published by

SPIE

Volume 11179

Part One of Two Parts 
The papers in this volume were part of the technical conference cited on the cover and title page. Papers were selected and subject to review by the editors and conference program committee. Some conference presentations may not be available for publication. Additional papers and presentation recordings may be available online in the SPIE Digital Library at SPIEDigitalLibrary.org.

The papers reflect the work and thoughts of the authors and are published herein as submitted. The publisher is not responsible for the validity of the information or for any outcomes resulting from reliance thereon.

Please use the following format to cite material from these proceedings:

Author(s), "Title of Paper," in Eleventh International Conference on Digital Image Processing (ICDIP 2019), edited by Jenq-Neng Hwang, Xudong Jiang, Proceedings of SPIE Vol. 11179 (SPIE, Bellingham, WA, 2019) Seven-digit Article CID Number.

ISSN: 0277-786X

ISSN: 1996-756X (electronic)

ISBN: 9781510630758

ISBN: 9781510630765 (electronic)

Published by

SPIE

P.O. Box 10, Bellingham, Washington 98227-0010 USA

Telephone +1 3606763290 (Pacific Time) · Fax +1 3606471445

SPIE.org

Copyright (c) 2019, Society of Photo-Optical Instrumentation Engineers.

Copying of material in this book for internal or personal use, or for the internal or personal use of specific clients, beyond the fair use provisions granted by the U.S. Copyright Law is authorized by SPIE subject to payment of copying fees. The Transactional Reporting Service base fee for this volume is $\$ 21.00$ per article (or portion thereof), which should be paid directly to the Copyright Clearance Center (CCC), 222 Rosewood Drive, Danvers, MA 01923. Payment may also be made electronically through CCC Online at copyright.com. Other copying for republication, resale, advertising or promotion, or any form of systematic or multiple reproduction of any material in this book is prohibited except with permission in writing from the publisher. The CCC fee code is $0277-$ $786 \mathrm{X} / 19 / \$ 21.00$.

Printed in the United States of America by Curran Associates, Inc., under license from SPIE.

Publication of record for individual papers is online in the SPIE Digital Library.

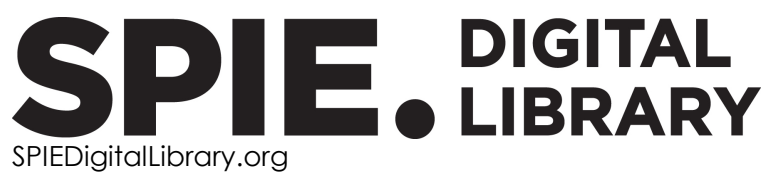

Paper Numbering: Proceedings of SPIE follow an e-First publication model. A unique citation identifier (CID) number is assigned to each article at the time of publication. Utilization of CIDs allows articles to be fully citable as soon as they are published online, and connects the same identifier to all online and print versions of the publication. SPIE uses a seven-digit CID article numbering system structured as follows:

- The first five digits correspond to the SPIE volume number.

- The last two digits indicate publication order within the volume using a Base 36 numbering system employing both numerals and letters. These two-number sets start with $00,01,02,03,04$, 05, 06, 07, 08, 09, OA, OB ... 0Z, followed by 10-1Z, 20-2Z, etc. The CID Number appears on each page of the manuscript. 


\title{
Contents
}

\author{
xiii Authors \\ xix Conference Committee \\ xxvii Introduction
}

\section{Part One}

IMAGE PROCESSING

1117902 Research on improving the authenticity of simulated infrared image using adversarial networks [1 $11179-4]$

1117903 Classification of blood cancer images using a convolutional neural networks ensemble [111179-6]

$1117904 \quad$ Algorithm of image de-noising for Gaussian-Gaussian mixed noise model based on stochastic resonance [1 $1179-21]$

$1117905 \quad$ Image steganography algorithm based on key matrix generated by quantum walks [1 $1179-25]$

$1117906 \quad$ Relative attitude estimation for small satellite based on stellar image matching [11179-31]

1117907 Semi-supervised semantic image segmentation using dual discriminator adversarial networks [1 11 179-54]

1117908 Remote sensing image fusion algorithm based on a trous wavelet transform and HIS transform [1 $11179-55]$

1117909 Content-based power allocation for perception-friendly SoftCast [1 $1179-87]$

$111790 \mathrm{~A} \quad$ Computer image analysis in evaluating the quality of dried meat, case study: poultry meat [11 1 179-91]

11179 OB Sparse 3D point clouds segmentation considering 2D image feature extraction with deep learning [1 $11179-92]$

11179 OC Health properties and evaluation of quality of dried strawberry fruit produced using the convective drying method with neural image analysis [1 $1179-95]$

11179 OD Anchored neighborhoods search based on global dictionary atoms for face photo-sketch synthesis [ $111179-103]$ 
11179 OE Velodyne LiDAR and monocular camera data fusion for depth map and 3D reconstruction [1 $1179-106]$

11179 OF Analysis of the effects of scrambling and diffusion of logistic chaotic map on image encryption [11 $1179-125]$

11179 OG Performance analysis of anti-noise interference of hemorrhagic cerebral stroke image reconstruction in chirp-pulse microwave CT (CP-MCT) [1 $11179-127]$

$11179 \mathrm{OH} \quad$ An accurate and efficient MR image reconstruction model [11179-162]

11179 Ol Image-to-image translation using a relativistic generative adversarial network [1 $1179-163]$

11179 0J BMIMatic: body mass index derivation from captured images [11 179-169]

11179 OK Sparse representation based medical ultrasound images denoising with reshaped-RED [11179-172]

11179 OL Improving resolution images detail features for Generate Network [1 $1179-179]$

11179 OM Research on blurred image restoration based on generative adversarial networks [11179-185]

11179 ON Generating large scale images using GANs [11 179-195]

1117900 A two-stage airport detection model on large scale SAR images based on faster R-CNN [1 $1179-200]$

11179 OP An improved segmentation method for porous transducer CT images [1 $1179-5]$

$111790 Q \quad$ A depth image reconstruction algorithm based on background noise censoring [11179-50]

11179 OR Fast segmentation of tea flowers based on color and region growth [1 $1179-65]$

11179 OS Image fusion methods based on compressed sensing: theory and application [11179-66]

11179 OT Single image super resolution based on generative adversarial networks [1 1179-69]

11179 OU Blind quality assessment of on-board reconnaissance images [11179-70]

11179 OV Accurate CT-ultrasound image registration using simulated transformation optimization [1 $11179-77]$

11179 OW Image watermarking algorithm based on quaternion and chaotic Lorenz system [11179-81]

11179 OX Image denoising and enhancement of terahertz passive imaging [1 $1179-112]$

11179 OY End-to-end image dehazing via dual principles of discriminativeness [1 $1179-120]$ 
$111790 Z$ Accurate single image super-resolution using cascading dense connections [1 $1179-139]$

1117910 Image denoising method and evaluation based on mixed wavelet algorithm [1 $1179-141]$

1117911 A method for single image phase unwrapping based on generative adversarial networks [11179-157]

1117912 Analysis of the effects of scrambling and diffusion of logistic chaotic map on image encryption [1 $1179-168]$

1117913 Image denoising based on non-subsampled shearlet transform use, non-local means, and hard threshold [111179-171]

1117914 An inquiry on image enhancement methods of remote sensing images [11179-176]

1117915 Single image super-resolution reconstruction based on multi-directionality of the edge [1 $1179-180]$

$1117916 \quad$ Medical image grading learning based on active and incremental fine-tuning [1 $1179-193]$

1117917 An image caption model incorporating high-level semantic features [11179-196]

1117918 An integration de-noising method based on self-adaptive manifold filtering and text contour for ancient Chinese calligraphy tablet image [1 $11179-204]$

1117919 The application of UAV remote sensing in natural disasters emergency monitoring and assessment [1 $11179-90]$

$111791 \mathrm{~A} \quad$ Fast image stitching of unmanned aerial vehicle remote sensing image based on SURF algorithm [1 11 179-209]

\section{PATTERN RECOGNITION}

$111791 \mathrm{~B} \quad$ Aerial infrared target recognition based on lightweight convolutional neural network [1 $1179-11$ ]

$111791 \mathrm{C} \quad$ Research on face detection method based on improved MTCNN network [1 $1179-16]$

11179 1D Novel algorithm for finger vein recognition based on inception-resnet module [11179-20]

$111791 \mathrm{E} \quad$ Neural classification of microscope digital pictures domestic pig oocytes [1 $1179-75]$

$111791 \mathrm{~F} \quad$ High-speed barcode recognition system based on OpenCV and Zbar [1 1179-84]

$1117916 \quad$ Vehicle color recognition based on superpixel features [1 1179-102] 
$111791 \mathrm{H} \quad$ Design of image recognition system based on OpenCL acceleration [1 $1179-110]$

$1117911 \quad$ Multi- perspective gesture recognition based on convolutional neural network [1 $1179-117]$

$111791 \mathrm{~J} \quad$ Study on segmentation and recognition algorithm of crystal in urinary sediment image [1 $11179-202]$

$111791 \mathrm{~K} \quad$ An ethnic costumes classification model with optimized learning rate [1 $1179-7]$

$111791 \mathrm{~L} \quad$ Skeleton based action recognition using pose change map and convolutional neural networks [1 $1179-28]$

$111791 \mathrm{M} \quad$ Palmprint recognition based on local joint edge and orientation patterns [1 $1179-36]$

$111791 \mathrm{~N} \quad$ Evaluating the potential of GF-1 pan-multispectral camera imagery for identifying the quasi-circular vegetation patches in the Yellow River Delta, China [1 $11179-41]$

$1117910 \quad$ Automatic recognition and correction of solar cells [1 $1179-43]$

$111791 \mathrm{P} \quad$ A parallel segmentation after classification algorithm of multi-spectral image of k-means of deep learning and panchromatic based on wavelet [1 $1179-47]$

$111791 Q \quad$ Face recognition method based on SVM and SRC [1 $1179-57]$

$111791 \mathrm{R} \quad$ Pattern recognition method for mobile intelligent programming learning [11179-88]

11179 1S Deep learning concepts and datasets for image recognition: overview 2019 [11179-100]

$111791 \mathrm{~T}$ Sequence recognition of natural scene house number based on convolutional neural network [1 $1179-107]$

$111791 \mathrm{U}$ Joint end-to-end learning for scale-adaptive person super-resolution and re-identification [1 $11179-122]$

$111791 \mathrm{~V} \quad$ A novel mice interaction recognition method by using machine learning [1 $1179-165]$

$111791 \mathrm{~W} \quad$ Real-time action recognition based on enhanced motion vector temporal segment network [11 $1179-173]$

$111791 \mathrm{X} \quad$ Micronucleus image recognition based on feature-map spatial transformation [11179-192]

$111791 \mathrm{Y} \quad$ Person re-identification algorithm based on the fusion of deep feature and LOMO feature [11 1179-199] 


\section{CLASSIFICATION AND DETECTION}

$1117912 \quad$ Examination paper text detection based on character discriminator [1 1179-17]

1117920 Airborne LiDAR point cloud classification based on transfer learning [1 $1179-22]$

$1117921 \quad$ Feature matching via guided motion field consensus [1 $1179-26]$

1117922 Initial investigation of different classifiers for plant leaf classification using multiple features [1 $11179-44]$

1117923 Internal and external similarity aggregation stereo match algorithm [1 $1179-62]$

$1117924 \quad$ Mapping and visualization of complex relational structures in the graph form using the Neo4j graph database [1 $11179-76]$

1117925 Automated detection of arousal event with fuzzy entropy using physiological signals [11179-1 16]

1117926 Dynamic vehicle target detection and traffic statistics algorithm research [1 $1179-136]$

1117927 An improved self-supervised framework for feature point detection [1 $1179-153]$

1117928 The small target detection based on local directional contrast associated with directional entropy [1 $11179-161]$

\section{Part Two}

1117929 Single shot relation detector for pedestrian detection [1 1179-181]

11179 2A Deinterlacing based on scene change and content characteristics detection [11179-187]

$111792 \mathrm{~B}$ Intraframe interpolation based on edge detection [11179-188]

11179 2C A new trademark detection method via trademark confidence score of MSERs [11179-59]

$111792 \mathrm{D} \quad$ Sequence image matching using adaptive SIFT under complex environmental conditions [1 $11179-63]$

$111792 \mathrm{E} \quad$ Double JPEG compression detection based on noise-free DCT coefficients histograms [11179-123]

$111792 \mathrm{~F} \quad$ Unsupervised facial image occlusion detection with deep autoencoder [11179-146]

$111792 \mathrm{G} \quad$ Adaptive edge detection via gray value differences of points [11179-210] 
$111792 \mathrm{H} \quad$ An active region corrected method for weakly supervised aircraft detection in remote sensing images [1 $11179-51]$

$1117921 \quad$ From simulation to reality: ground vehicle detection in aerial imagery based on deep learning [1 $1179-83]$

$111792 \mathrm{~J} \quad$ Accurate features point extraction for omnidirectional camera calibration [1 $1179-108]$

11179 2K Research on improved image registration algorithm based on PROSAC algorithm [1 $1179-111$ ]

$111792 \mathrm{~L} \quad$ Multi-target tracking of surveillance video with differential YOLO and DeepSort [1 1179-174]

$111792 \mathrm{M}$ An objective assessment method for video stabilization performance [11179-189]

$111792 \mathrm{~N} \quad$ Accurate scale estimation for correlation filter based visual tracking [1 $1179-24]$

$1117920 \quad$ HEVC fast fractional-pixel prediction with parallel search [1 11 179-35]

11179 2P A tasseled cap transformation for CBERS-04 fusion multispectral images [1 $1179-39]$

$111792 \mathrm{Q}$ Feasible region division of panoramic images based on morphological filter [1 $1179-46]$

$111792 R \quad$ Stabilization of atmospheric turbulence-distorted video using complex steerable pyramid [11179-49]

$1117925 \quad$ A new method of visualizing the road traffic: differential timing method [11179-73]

$111792 \mathrm{~T} \quad$ Video frames encryption based on DNA sequences and chaos [1 1179-129]

$111792 \mathrm{U} \quad$ Remote sensing image texture enhancement based on HSV-BEMD algorithm [11179-142]

$111792 \mathrm{~V} \quad$ Visual object tracking based on adaptive multi-feature fusion in complex scenarios [11179-143]

11179 2W High-quality hybrid imaging with a cubic phase mask [11179-149]

$111792 X \quad$ Rendering circular depth of field effect with integral image [11179-158]

$111792 Y \quad$ Fast inter-frame coding algorithm for 360-degree videos [1 1179-184]

$1117922 \quad$ Digital video stabilization based on block motion estimation and iterative optimization of GMP [11179-190] 
$1117930 \quad$ Learning saliently temporal-spatial features for x-ray coronary angiography sequence segmentation [1 $11179-2]$

$1117931 \quad$ Oil slick extraction from hyperspectral images using a modified stacked auto-encoder network [1 $1179-52]$

1117932 Research on vehicle license plate data generation in complex environment based on generative adversarial network [1 $1179-105]$

1117933 Hyperspectral unmixing using double reweighted collaborative sparse regression [1 $1179-140]$

1117934 Convolutional-neural-network-based feature extraction for liver segmentation from CT images [11 1179-159]

1117935 A novel fusion strategy for probabilistic sparse representation classifier guided by support vector machines [1 $11179-34]$

1117936 Deep transfer learning for MR image feature point descriptors [1 $1179-53]$

1117937 An enhanced non local mean method for hole filling in the depth image-based rendering system [1 $1179-82]$

1117938 Fashion popularity analysis based on online social network via deep learning [1 $1179-89]$

1117939 Identification of co-substrate composted with sewage sludge using convolutional neural networks [1 $11179-99]$

11179 3A The cache construction and intelligent imagination of airfare shopping engine [11179-121]

\section{DATA MINING}

11179 3B Video question answering by frame attention [11179-14]

$111793 \mathrm{C}$ Scatter kernel parameters optimization for digital radiography [1 $1179-29]$

11179 3D A new approach to robust fundamental matrix estimation using an analytic objective function and adjusted gradient projection [1 $11179-38$ ]

$111793 \mathrm{E} \quad$ On the target area tracking method for heart rate measurement using deep learning strategy [11 179-78]

11179 3F The method and a stand for measuring aerodynamic forces in every plane on the basis of an image analysis [1 $11179-118]$

$1117936 \quad$ Efficient level set formulation for segmentation and correction with application to medical images [1 $11179-138]$ 
$111793 \mathrm{H} \quad$ Adaptive fractional-order differentiation filter guided by feature asymmetry for featurepreserving ultrasound despeckling [1 $11179-144]$

$1117931 \quad$ The system of motion capture based on inertial sensor [11179-154]

11179 3J The comparative analysis of Chinese GDP spatialization methods based on multi-sensor remote sensing data [1 $11179-23]$

$111793 \mathrm{~K} \quad$ A robust anti-occlusion object tracking method [11179-33]

$111793 \mathrm{~L} \quad$ An improved blind watermarking method based on SWT and LU decomposition [11179-79]

$111793 \mathrm{M} \quad$ Real-time oxygen saturation measurement based on reflective region removal technology [1 11 179-98]

$111793 \mathrm{~N} \quad$ An adversarial approach to non-uniform blind deblurring with opposite-channel-based discriminative priors [11 179-101]

1117930 The validation of the method of speed test of seeds moving in a tube of a pneumatic seed drill [1 $1179-119]$

11179 3P Singular value decomposition compressed ghost imaging based on non-negative constraints [1 $1179-170]$

$111793 Q$ 3D face landmarking with denoise auto-encoder networks [11179-198]

DATA ANALYSIS

$111793 R \quad$ Uncovering vein pattern using generative adversarial network [1 $1179-3$ ]

1117935 A stereo matching network with a cascade spatial pyramid pooling (CSPP) substructure [1 $11179-12]$

11179 3T Adversarial training and dilated convolutions for compressed sensing MRI [11179-19]

$111793 \mathrm{U} \quad$ High-resolution imaging of space target based on compressed sensing [11179-37]

11179 3V Automatic detection of solar radio burst using k-means clustering [1 $1179-56]$

$111793 \mathrm{~W} \quad$ Advantages and disadvantages of typical visual saliency methods applied to ship detection on sea surface [1 $1179-58]$

$111793 X \quad$ A survey of data hiding schemes based on AMBTC [11179-74]

11179 3Y 1-bit compressed sensing based on reweighting approximate message passing [11179-80] 
1117932 A high-speed multi-scale kernel correlation filter tracking algorithm [1 $1179-86]$

$1117940 \quad$ Application of neural modelling methods in the evaluation of the quality of pork half-carcasses [1 11 179-93]

$1117941 \quad$ Neural image analysis in determining the content of dry matter in corn cob [11179-94]

1117942 The selection of a representative substance for image analysis assessment [1 $1179-97$ ]

1117943 Pose estimation of a single circle based on 1D homography [11179-109]

1117944 A viterbi algorithm-based IF estimator for overlapped multicomponent signal [11179-131]

$1117945 \quad$ YOLOv3-based face detector with focal loss [11179-132]

1117946 Research on nonlinear correction system of current transformer [11179-134]

1117947 Image feature point extraction based on neural network is implemented on FPGA [11 179-148]

1117948 Mono-camera based calibration method for two-axes LRF measurement system [11179-166]

$1117949 \quad$ Mixed 3D-(2+1)D convolution for action recognition [1 $11179-177]$

11179 4A Recent development of automotive LiDAR technology, industry and trends [1 $1179-178]$

$111794 \mathrm{~B} \quad$ A novel proximity algorithm for an unconstrained convex segmentation model [11179-183]

11179 4C An automatic detection method of solar radio burst based on Otsu binarization [11179-30]

$111794 \mathrm{D} \quad$ A framework for the revision of large-scale image retrieval benchmarks [1 $1179-32]$

$111794 \mathrm{E} \quad$ Shape correspondence based effective combination of linear and quadratic assignment matrices [1 $11179-42]$

$111794 \mathrm{~F} \quad$ Contrast enhancement for the detection of stroke signs in CT using PCA based image fusion [1 $1179-48]$

$111794 \mathrm{G} \quad$ Registration between UAV LiDAR datasets for under-forest areas [1 $1179-67]$

$111794 \mathrm{H} \quad$ A model based method for stereo face reconstruction [1 $1179-71]$

$1117941 \quad$ Design and implementation of automatic aiming system based on YOLO [1 1179-72]

$111794 \mathrm{~J} \quad$ An efficient FCN based neural network for image semantic segmentation [1 $1179-147]$ 
$111794 \mathrm{~K} \quad$ Vertebrae segmentation via stacked sparse autoencoder from computed tomography images [1 $1179-160]$

$111794 \mathrm{~L} \quad$ 3D rigid pose tracking based on new distance function of line segments [1 1179-167]

$111794 \mathrm{M} \quad$ Research on single channel Doppler direction finding system [1 1179-182]

$111794 \mathrm{~N} \quad$ Survey on block-based motion estimation for frame rate conversion [11179-186]

1117940 Multi-channel compressed sensing optimization based on singular value decomposition [11179-191]

$111794 \mathrm{P} \quad$ Single image super-resolution based on gradient profile prior and nonlocal self-similarity feature [1 $11179-211]$ 


\section{Authors}

Numbers in the index correspond to the last two digits of the seven-digit citation identifier (CID) article numbering system used in Proceedings of SPIE. The first five digits reflect the volume number. Base 36 numbering is employed for the last two digits and indicates the order of articles within the volume. Numbers start with 00, 01, 02, 03, 04, 05, 06, 07, 08, 09, OA, 0B...0Z, followed by 10-1Z, 20-2Z, etc.

\author{
A., Sukeshkumar, $4 \mathrm{~F}$ \\ Abd El-Atty, Bassem, OW \\ Abd El-Latif, Ahmed A., 05, OW \\ Agrawal, Somya, $3 \mathrm{X}$ \\ Ahmad, Mubashir, 34, 4K \\ Ai, Danni, OV, 4K \\ Akhtar, M. Rayyan, OE \\ Bai, Junfeng, 29 \\ Bai, Xue, IW \\ Bai, Yujun, 4M \\ Bao, Bing-Kun, OY, 3N \\ Boniecki, P., OA, OC, 1E, 24, 39, 40, 41, 42 \\ Brekhna, Brekhna, 2G \\ Cai, Limin, $1 \mathrm{H}$ \\ Cao, Ziyang, $3 \mathrm{~J}$ \\ Cardino, Adrian Christopher, $0 \mathrm{~J}$ \\ Chang, Chin-Chen, $3 \mathrm{X}$ \\ Chang, Wen, 31 \\ Chao, Yuyan, 18 \\ Chen, Chuan, 3A \\ Chen, Enqing, IW \\ Chen, Fuqu, 1J \\ Chen, Guancheng, OE \\ Chen, Jia, 36 \\ Chen, Jiahui, 2D \\ Chen, Jianxin, 04 \\ Chen, Junyu, 14 \\ Chen, Qian, $0 Q$ \\ Chen, Qianwen, 3P, 40 \\ Chen, Yupeng, 3M \\ Chen, Yuxiang, $1 \mathrm{M}$ \\ Cheng, Lei, 20 \\ Cheng, Xuelong, 3A \\ Cheng, Yinbo, 3Y \\ Chi, Jianning, $1 Y$ \\ Cong, Weijian, OV \\ Cui, Dongliang, $\mathrm{OZ}$ \\ Cui, Tingting, 1B \\ Cui, Zexiao, 3V \\ Deng, Hui, OS \\ Deng, Jiamei, 16, 17, 1X \\ Deng, Xiangyang, 11 \\ Ding, Yijun, 1D \\ Ding, Yuan, 34 \\ Dong, Gaojie, 31 \\ Dong, Jiahui, OV \\ Dong, Liang, 3V, 4C \\ Dong, Liquan, 2W \\ Du, Cuibing, 3D
}

Du, Yanxia, 4B

Duda, A., OC

El-Atty, Bassem Abd, 05

Fan, Jingfan, OV

Fan, Jinghua, $O M$

Fan, Qiang, 3K

Fan, Xuewu, 3U

Fang, Guoxin, $1 T$

Fang, Jiannan, 3B

Fang, Yan, 4P

Feng, G., $2 \mathrm{U}$

Feng, Guoqi, $\mathrm{OZ}$

Feng, Liangjie, 08

Fernandez, Clarence, 0J

Frankowski, Jakub, 0C, 24, 42

Fu, Bin, $3 Z$

Fu, Chunlan, 1M

Fu, Qinglin, 47

Gai, Shaoyan, 3Q

Gan, Zongliang, OD

Gao, Chao, 2F

Gao, Chongyang, 1Q

Gao, Guannan, 3V, 4C

Gao, Jing, $1 T$

Gao, Shaoshuai, 09

Gao, Wei, OK, $3 Z$

Gao, Yun, 3V, 4C

Gawałek, J., 0A

Ge, Bailin, 3E, 3M

Ge, Feihang, 18

Ge, Jianhui, OL, 13

Ge, Qi, 1U, 3N

Ge, Rui, $3 U$

Gierz, Ł., OA, OC, 1E, 24, 39, 3F, 3O, 40, 41, 42

Gong, Qinghai, $2 Q$

Gong, Xuanrui, 2J

Gu, Guohua, OQ

Guan, Zhenzhi, IR

Guo, Biao, 1V

Guo, Ling, 41

Guo, Ruoyu, OP

Guo, Shaojie, 3V, 4C

Guo, Shuai, 3Q

Guo, Xiwei, 06, 2N

Han, Dong, 06, 2N

Han, Yu, 48

Hao, Dongdong, 30, 3H

Hao, Xiangyang, $2 \mathrm{~L}$

Hao, Youfei, 2D 
Hassan, Muhammad Umair, 4D, 4E

He, Lifeng, 18

$\mathrm{He}$, Peng, 06

He, Ruhan, 36

$\mathrm{He}$, Weiji, $0 \mathrm{Q}$

He, Ying, 1D

Heng, Yong, 1B

Hong, Luning, 26

Horak, Karel, 15

Hou, Boxiang, $1 \mathrm{~L}$

Hu, Jiwei, 16, 17, $1 \mathrm{X}$

$\mathrm{Hu}$, Jun, 2L

Hu, Qingshuang, OF, 12

$\mathrm{Hu}, \mathrm{TaO}, 1 \mathrm{~J}$

$\mathrm{Hu}$, Xinrong, 36

$\mathrm{Hu}$, Yong-jiang, OS

Hua, Bei, 07

Hua, Ruzhao, $1 Y$

Huang, Bin, 1L

Huang, Jianqiang, OT

Huang, Jintao, 19, 2K, 32

Huang, Pengwei, $O Q$

Huang, Qian, 2A, 2B, 2M, 2Z, 4N

Huang, Rui-yang, 2F

Huang, Yuan, 2A, 2B

Hui, Mei, 2W

Idziaszek, P., 24, 42

llao, Adomar L., 0J

Jia, Jinfang, OT

Jia, Wenjing, $3 G$

Jiang, Haiyang, 36

Jiang, Hao, 4A

Jiang, Jianpeng, $O M$

Jiang, Zhaoguo, 2J

Jiao, Mengyu, 09

Jin, Menglin, 4C

Jin, Peiquan, $2 \mathrm{H}$

Kainat, Wajiha, 3T

Khalifa, Hany S., 05, 0W

Kong, Lingqin, 2W

Koszela, K., OA, 0C, 1E, 24, 39, 30, 40, 41, 42

Kuang, Gangyao, 00

Kujawa, S., OA, OC, 1E, 24, 39, 40, 41, 42

Kuzimska, T., 1E

Lam, Choi Ut, $2 S$

Lee, Chin-Feng, $3 \mathrm{X}$

Lei, Bo, 3K

Lei, Hao, OK

Lei, Tianjie, 19, 1A, 2K, 32

Li, Baopeng, OK

Li, Chenghai, OF, 12

Li, Chuyin, 00

Li, Cong, 11

Li, Congli, OU

Li, Daoji, 20

Li, Dongdong, 11

Li, Guan-Long, 3X

Li, Hai-Bo, OY, 1U, 3N

$\mathrm{Li}$, Jingsong, $2 \mathrm{~W}$

Li, Jun, 1T, 27
$\mathrm{Li}$, Jun, 31,47

$\mathrm{Li}, \mathrm{Kai}$, OT

Li, Lin, 3C

$\mathrm{Li}, \mathrm{Na}, 4 \mathrm{E}$

Li, Po, 44

Li, Qing, 2C

Li, Runwen, $4 \mathrm{M}$

Li, Shao-mei, $2 \mathrm{~F}$

Li, Shican, 19, 1 A

Li, Shuai, 10

Li, Shuo-Guo, OR

Li, Sijie, OG

Li, Weibin, 4B

Li, Xiangyu, 19, 2K

$\mathrm{Li}$, Xiaoguang, $1 \mathrm{Z}$

Li, Xuejian, 02, 2I, 4J

Li, Xuemei, 2G, 4P

Li, Yan, 33

$\mathrm{Li}$, Yanping, 2A, 2B

Li, Yashang, OX, 10

Li, Yuesong, 13, 15

$\mathrm{Li}$, Yusheng, OB

$\mathrm{Li}$, Zhenzhen, 1B

$\mathrm{Li}, \mathrm{Zhi}, 3 \mathrm{~W}$

Li, Zhixin, OK

Liang, Xiaohui, OV

Liang, Zi, $1 Y$

Liao, Lihua, 1Q

Liao, Qingmin, 25, 29, 3C, 3D, 3S, 45, 4H

Lin, Qiuli, OD, $1 G$

Lisiak, D., 40

Liv, Beibei, 07

Liu, Bingxin, 31

Liu, Changyuan, 26

Liu, Chen, OF, 12

Liu, Feng, OD, $1 G$

Liu, Fuchang, $2 X$

Liv, Heng, $0 G$

Liu, Hui, $1 \mathrm{~J}$

Liu, Jie, 2C, 46

Liu, Jing, OL, 13, 15, 4M

Liu, Jiwei, OK, 14, 32

Liu, Junping, 36

Liu, Junqi, 3W

Liu, Min, OM

Liu, Ming, 2W

Liu, Mingjun, 4E

Liu, Qingsheng, 1N, 2P

Liu, Quan, 16, 17, 1X

Liu, Songlin, 2L

Liu, Wei, 43

Liv, Xiao, 20

Liu, Xiaohua, 2W

Liu, Xiaotong, $1 \mathrm{~J}$

Liu, Xuemei, 19, 1A, 32

Liu, Xueshi, 2Q

Liv, Yi, 2G, 4P

Liu, Yifu, $1 R$

Liu, Yiming, 30

Liv, Yizhang, 21 
Liu, Yuan-Yuan, OY, 3N

Liu, Zhiyuan, 21

Liu, Zongwei, 4A

LU, Chun, 3A

Lu, Wenjun, OU

Lu, Zongqing, 25, 29, 3C, 3D, 3S, 4H

Luo, Guibo, 1V

Luo, Wuyi, $2 Q$

Luo, Zhiwang, 17

LV, Hui, 4I

LV, Yao-Wen, 2J, 43

Ma, Gehua, 3R

Ma, Honglin, 4B

$\mathrm{Ma}$, Kaiqiang, 03

$\mathrm{Ma}, \mathrm{Ke}, 2 \mathrm{G}$

Ma, M., $2 \mathrm{U}$

Ma, Xiaohu, $3 \mathrm{~L}$

Marfia, Gustavo, 38

Mazurkiewicz, J., 39

Mei, Kunqiang, 3H

Miao, LiGang, 10

Ming, Li, OP

Mohsen, Mohamed, ON

Moustafa, Mohamed, ON

Mu, Chengpo, 02, 2l, 4J

Mueller, W., OA, OC, 1E, 24, 39, 40, 41

Nan, Jinghong, 1B

Ning, $\mathrm{Ke}, \mathrm{OP}$

Niu, Dongmei, 4D, 4E

Niu, Xiaotong, 2E

Pan, Xiong, 21

Pan, XU, IV

Pau, Giovanni, 38

Pei, Jie, 46

Peng, Jialiang, 05, 0W

Peng, Jinxi, IP

Peng, Yanhong, $2 X$

Pilarska, A., OC

Przybył, J., OA, 41, 42

Przybył, K., 0A, 0C, 1E, 24, 39, 40, 41, 42

Pu, Xiaoqiu, OK, 14

Pu, Yuanyuan, 1C

Qadri, Syed Furqan, 34, 4K

Qian, Pengfei, $2 Y$

Qin, Binjie, 30, 3H

Qin, Huabiao, OE

Qin, Jing, 32

Qin, XuXu, OH

Qu, Zhiyong, $1 \mathrm{~F}$

R. S., Jeena, 4F

Ren, Yuyan, 26

Sablatnig, Robert, is

Saulon, Lawrence, 0J

Semkło, Ł., 42

Shao, Wen-Ze, OY, 1U, 3N

Shaukat, Kamran, 4D

Shen, Junge, 2E

Shen, Yanchun, 10

Shi, Miaowen, 4P

Shilu, S. L., 4F
Shohag, Md Shakil Ahamed, 4D

Shu, Xiu, 3G

Si, Jingjing, $3 Y$

Ślósarz, P., 40

Song, Chao, 4B

Song, Donghong, $1 \mathrm{P}$

Song, Jiazhong, 04

Song, Zongxi, 14, 1K, $3 Z$

Staszak, Ż., 30

Su, Yuanqi, $1 \mathrm{P}$

Su, Zhuo, 16

Sun, Lingling, 03, 3B

Sun, Sidong, $2 M, 2 Z$

Tan, Hai, 3K

Tang, Chaoying, 3R

Tang, Jun, 3P, $4 \mathrm{O}$

Tang, Linbo, 1B

Tang, Su-Kit, $2 \mathrm{~S}$

Tang, Ting, $3 \mathrm{~S}$

Tang, Wenhai, 25

Tao, Jinxu, 3T

Tao, Liang, 1D, 1M

Tao, Wang, 4G

Tian, Guohui, $1 \mathrm{~L}$

Tian, Jiandong, OB, 11

Tian, Qijun, 2H

Tian, Yong, OB, 11

Tinega, Haron Chweya, IW

Tse, Rita, 2S, 38

Tu, Rongxin, 2T

Wan, Shouhong, $2 \mathrm{H}$

Wan, Yan, OM

Wang, Biao, 3R

Wang, Chang-long, os

Wang, Feng, 3 Z

Wang, Heng, $1 \mathrm{H}$

Wang, Hengjun, 2V

Wang, Huabin, 1D, 1M

Wang, Jiabao, 19, 2K, 32

Wang, Jian, OR

Wang, Jiedong, 48, 4L

Wang, Jing, 3U

Wang, Junchao, 03

Wang, Junqiang, 2L

Wang, Liang, $3 Q$

Wang, Linteng, $1 Z$

Wang, Li-Qian, OY, $1 \mathrm{U}$

Wang, Meiling, $O P$

Wang, Meiqin, 3P, $4 \mathrm{O}$

Wang, Min, 3V, 4C

Wang, Shengqian, 33

Wang, Wei, $1 \mathrm{~J}$

Wang, Wenhao, $1 Y$

Wang, Xian, 1D

Wang, Xiaoying, OT

Wang, Xu-dong, $2 F$

Wang, Yanfang, 2A, 2B, 2M, 2Z, $4 \mathrm{~N}$

Wang, Yang, $1 \mathrm{C}$

Wang, Yanjie, 02

Wang, Yaqi, 03, 3B 
Wang, Yiming, $2 \mathrm{M}, 2 \mathrm{Z}, 4 \mathrm{~N}$

Wang, Yongtian, 4K

Wang, Yuanqing, 37

Wang, Zhenwei, 28

Wei, Hong-quan, 2F

Wei, Kangkang, 2T

Wei, Wei, 0 Z

Wen, Desheng, 14

Wen, Wenying, 2T

Wojcieszak, D., 0C, 1E, 24, 39, 30, 41

Wu, Hao, $1 \mathrm{C}$

Wu, Jie, 3L

Wu, Yunhui, 27

Wu, Zhifeng, 3J

Xia, Tengxi, IR

Xiang, Guoqing, 20

Xiao, Lihua, 48, 4L

Xiao, Wenjuan, 41

Xie, Shi-Peng, $1 \mathrm{U}$

Xin, Hongqiang, 08

Xing, Xingrun, $\mathrm{Ol}$

$\mathrm{Xu}$, Baohua, 2N

$\mathrm{Xu}, \mathrm{Chao}, 3 \mathrm{~T}$

$X U$, Dan, $1 C$

$\mathrm{Xu}$, Hengwei, $4 \mathrm{H}$

$\mathrm{XU}$, Jian, $2 \mathrm{H}$

$X U$, Jiawei, 3A

$X U$, Jing, 32

$X U$, Jinzhang, $3 T$

$X U$, Jiwei, $2 L$

$X U$, Juan, $1 \mathrm{U}$

$X u$, Junfeng, 20

$X \cup, P e i, 3 Y$

$X U$, Ran, OD, $1 G$

$X u, X i-P e n g, 43$

$\mathrm{Xu}$, Yonghui, $1 \mathrm{~F}$

$X \cup, Y u j i e, 1 X$

$X \mathrm{U}$, Zicheng, $2 \mathrm{~J}, 43$

Xue, Bindang, $2 R$

Xue, Jing-Hao, 3D

Xue, Rui, OL, 15

Xue, Song, OU

Xue, Xiaorong, $1 \mathrm{P}$

Xue, Xiaoyong, IP

Yan, Chunman, 2D

Yan, Wei, 20

Yan, Yunyao, 20

Yang, Bin, 49

Yang, Changcai, 21

Yang, Cheng, OR

Yang, Dongsheng, 23

Yang, Jian, OV, 34

Yang, Li, $1 \mathrm{~J}$

Yang, Ping, 4M

Yang, Ruixin, 2l, 4J

Yang, Shenghao, OT

Yang, Wenming, 45

Yang, Xin, 10

Yang, Yang, $2 X$

Yang, Yingbin, $1 \mathrm{H}$
Yang, Yu, 02, 2l, 4J

Yang, Yunna, $\mathrm{OH}$

Yang, Yunyun, $\mathrm{OH}, 3 \mathrm{G}$

Yang, Zhengbo, 4M

Yao, Li, OM

Ye, Feng, 2A, 2B, 2M, 2Z, 4N

$Y e$, Jiaquan, $4 M$

Ye, Liang, OT

Ye, Lu-Yue, OY, 3N

Ye, Zhongfu, 3T

Yi, Xian, 4B

Yin, Jian, 45

Yu, Bo, $1 \mathrm{R}$

Yu, Congcong, 3U

Yu, Donghang, 20

YU, Han, 4L

Yuan, Guowu, 1C, 3V, 4C

Yuan, Man, 1A, 2K, 32

Zaborowicz, M., OA, 1E, 39, 40, 41

Zeng, Lanling, $2 X$

Zeng, Shaoning, 22, 35

Zhai, You, 06, 2N

Zhan, Lingli, 37

Zhang, Baoming, 20

Zhang, Bob, 22, 35

Zhang, Chao, $2 \mathrm{R}$

Zhang, Cheng, 3P, $4 \mathrm{O}$

Zhang, Dawei, ol

Zhang, Di, 2D, 4N

Zhang, Dongdong, $2 Y$

Zhang, Haiying, 28

Zhang, Jie, OF, 12

Zhang, Limin, 11

Zhang, Ling, 3U

Zhang, Mingxuan, 4D, 4E

Zhang, Qi, 22

Zhang, Qiang, 31

Zhang, Ruiheng, 02, 21

Zhang, Sai, 3E

Zhang, Shengbo, 10

Zhang, Shuang, 1K, 3 Z

Zhang, Shuwu, 2C

Zhang, $\mathrm{XU}, 2 \mathrm{~L}$

Zhang, Xueyang, 3W

Zhang, $Y \cup, 04$

Zhang, Yuan, $2 \mathrm{C}$

Zhang, Yue, $1 Y$

Zhang, Yu-hua, OS

Zhang, Zejun, 21

Zhang, Zhan, 23

Zhang, Zihao, 37

Zhao, Chuan, 20

Zhao, Chun, 2K

Zhao, Fangzheng, OF, 12

Zhao, Fuquan, 4A

Zhao, Guozhong, 0X, 10

Zhao, Hui, $3 \mathrm{U}$

Zhao, Huimin, $1 \mathrm{~T}$

Zhao, Lingjun, 00

Zhao, Qiang, $1 G$ 
Zhao, Wenshuang, 4D

Zhao, Xiuyang, 4D, 4E

Zhao, Yalan, 3J

Zhao, Yan, $2 X$

Zhao, Yingbin, OM

Zhao, Yuejin, $2 \mathrm{~W}$

Zhao, Zhiqi, 4K

Zheng, Dong, $1 \mathrm{C}$

Zheng, Huihuang, 28

Zheng, Jieying, OD

Zheng, Shutao, $1 \mathrm{~F}$

Zheng, Yang, $2 \mathrm{C}$

Zhong, Jiachen, 38

Zhong, Juping, $1 T$

Zhong, Yan-Zhen, $1 \mathrm{U}$

Zhou, Fugen, 2R

Zhou, Hao, 3V, 4C

Zhou, Jianhang, 35

Zhou, Jin, 4E

Zhou, Langming, 48, 4L

Zhou, Ping, 49

Zhou, Zhongjun, 28

Zhu, Hongjuan, 1Q

Zhu, Nan, 2E

Zhu, Ni, 3A

Zhu, Xuan, 46

Zhu, Yuanyuan, 3P, 40

Zhu, Yuesheng, $1 \mathrm{~V}$

Zong, Shouxin, 09

Zou, Jiancheng, 3E, 3M

Proc. of SPIE Vol. 11179 1117901-17 
Proc. of SPIE Vol. 11179 1117901-18

Downloaded From: https://www.spiedigitallibrary.org/conference-proceedings-of-spie on 26 Apr 2023 Terms of Use: https://www.spiedigitallibrary.org/terms-of-use 


\title{
Conference Committee
}

\author{
Honorary Chairs \\ Yongqi Xue, Shanghai Institute of Technical Physics, CAS (China) \\ International Advisory Committee \\ Josiane Zerubia, IEEE Fellow, INRIA (France) \\ Jón Atli Benedikłsson, University of Iceland (Iceland) \\ Conference Chairs \\ Jun Li, Sun Yat-Sen University (China) \\ Weishi Zheng, Sun Yat-Sen University (China) \\ Jenq-Neng Hwang, University of Washington (United States) \\ Xudong Jiang, Nanyang Technological University (Singapore) \\ Program Committee Chairs \\ Qingli Li, East China Normal University (China) \\ Christine Fernandez-Maloigne, Université Poitiers (France) \\ Yong Wang, Harbin Institute of Technology (China) \\ Wenbing Tao, Huazhong University of Science and Technology (China) \\ Steering Committee \\ Yuri Rzhanov, University of New Hampshire (United States) \\ Xiaobo Lu, Southeast University (China) \\ Publicity Chairs \\ Giovanni Pau, Université Pierre et Marie Curie (France) \\ András Horváth, Peter Pazmany Catholic University (Hungary) \\ Krzysztof Koszela, Poznan University of Life Sciences (Poland)

\section{Publication Chair}

Yi Xie, Wuhan University (China) 
Technical Committee

Yang Xin, Beijing University of Post and Telecommunications (China) Liming Zhang, University of Macau (Macao, China) Jinfeng Yang, Civil Aviation University of China (China) Yong-Sheng Chen, National Chiao Tung University (Taiwan, China) Tarek Sobh, University of Bridgeport (United States) Mueller Wojciech, Poznan University of Life Sciences (Poland) Srikanta Murthy, PES School of Engineering (India) Radosław Jan Kozłowski, Poznan University of Life Sciences (Poland) Gniewko Niedbała, Poznan University of Life Sciences (Poland) Bicheng Li, Information Engineering University (China) Lixiong Liu, Beijing Institute of Technology (China) Fulin Su, Harbin Institute of Technology (China)

Zhi Liu, Shanghai University (China)

Bin Tang, University of Electronic Science and Technology of China (China)

Xiaoyong Lei, Beihang University (China)

En-Bing Lin, Central Michigan University (United States)

Huimin Ma, Tsinghua University (China)

Juncheng Li, Hunan University of Humanities, Science, and Technology (China)

Mingzhe Liu, Chengdu University of Technology (China)

Muhammad Naufal Bin Mansor, Universiti Malaysia Perlis (Malaysia)

George A. Papakostas, Eastern Macedonia and Thrace Institute of Technology (Greece)

Zhi Jia Zhang, Shenyang University of Technology (China)

Tianqing Peng, Henan Institute of Engineering (China)

Tieling Chen, University of South Carolina, Aiken (United States)

Hong Lu, Nanjing Institute of Technology (China)

Florence Cloppet, Université Paris Descartes (France)

Momina Moetesum, Bahria University (Pakistan)

Imran Siddiqi, Bahria University (Pakistan)

Bin Yan, National Digital Switching System Engineering and

Technological Research Center (China)

Wu Xi, Xihua University (China)

Wu-Hsiung Chen, Pano Leader Company, Ltd. (Taiwan, China)

Hengjian Li, Shandong Computer Science Center (China)

Sergey Kravtsov, Southern Federal University (Russian Federation)

Konstantin Rumyantsev, Southern Federal University (Russian Federation)

Yan Yang, Southwest Jiaotong University (China)

Sherif Welsen, University of Nottingham Ningbo (China)

Ningyu Zhang, Shandong Jianzhu University (China)

Chunning Meng, China Maritime Police Academy (China)

Jenq-Neng Hwang, University of Washington (United States)

Hua-Tsung Chen, National Chiao Tung University (Taiwan, China)

Ahmed A. Abd El-Latif, Menoufia University (Egypt)

Mark Richard Pickering, The University of New South Wales (Australia) 
Hongping Li, Ocean University of China (China)

Huiqin Jiang, Zhengzhou University (China)

Tao Wu, Lingnan Normal University (China)

Kathiravan Srinivasan, National llian University (Taiwan, China)

Liu Zhen, National University of Defense Technology (China)

Yigang Zhou, Harbin Institute of Technology (China)

Xibin Jia, Beijing University of Technology (China)

Fengqi Li, Dalian University of Technology (China)

Nicole Vincent, Université Paris Descartes (France)

Ahmed Nashat, Fayoum University (Egypt)

Zhihua Xie, Jiangxi Science and Technology Normal University (China)

Kuo-Liang Chung, National Taiwan University of Science and Technology (Taiwan, China)

G. Balakrishnan, Indra Ganesan College of Engineering (India)

Peng Wang, Tsinghua University (China)

Juan Li, Beijing Jiaotong University (China)

Jing Hu, Chengdu University of Information and Technology (China)

Hung Nguyen, Japan Advanced Institute of Science and Technology (Japan)

Yangming He, Jiangxi University of Traditional Chinese Medicine (China)

Bing Li, State Key Laboratory of Complex Electromagnetic Environmental Effects on Electronics and Information Systems (China)

Fei Xia, Shanghai University of Electric Power (China)

Lifeng Zhang, North China Electric Power University (China)

Dongming Zhou, Yunnan University (China)

Junzhou Zou, East China University of Science and Technology (China)

Wenchao Cui, China Three Gorges University (China)

Hong Zhang, Armstrong State University (United States)

Suyu Wang, Beijing University of Technology (China)

Shua Lu, Jilin University (China)

Dongmei Fu, University of Science and Technology Beijing (China)

Yangming He, Jiangxi University of Traditional Chinese Medicine (China)

Peiyuan Guo, Beijing Technology and Business University (China)

Albert Chong, University of Southern Queensland (Australia)

Yebin Liu, Tsinghua University (China)

Lizhuang Ma, Shanghai Jiao Tong University (China)

Hongtao Xie, Institute of Information Engineering, CAS (China)

Tao Lei, Institute of Optics and Electronics, CAS (China)

Wen He, Chengdu Medical College (China)

Chi-Man Pun, University of Macau (Macao, China)

Zhen Liu, Ningbo University (China)

Guowang Jin, Zhengzhou Institute of Surveying and Mapping (China)

Hu Zheng, National University of Defense Technology (China)

Xiangyang Hao, Information Engineering University (China)

Maciej Zaborowicz, Poznan University of Life Sciences (Poland)

Yusnaidi Md Yusof, Universiti Teknologi Malaysia (Malaysia)

Kin Hong Wong, The Chinese University of Hong Kong (Hong Kong, China) 
Ruofei Zhong, Capital Normal University (China)

Kaixia Wei, Nanjing Xiaozhuang University (China)

Zhenzhou Wang, Shenyang Institute of Automation, CAS (China)

Zhongjun Zhang, Beijing Normal University (China)

Yongqi Sun, Beijing Jiaotong University (China)

Yan-Guo Wang, China Academy of Railway Sciences (China)

Wangmeng Zuo, Harbin Institute of Technology (China)

Manhua Liu, Shanghai Jiao Tong University (China)

Fan Zhao, Xi' an University of Technology (China)

Zhenghao Shi, Xi' an University of Technology (China)

Linbo Qing, Sichuan University (China)

Changwen Zheng, University of Chinese Academy of Sciences (China)

Wen-Jye Shyr, National Changhua University of Education

(Taiwan, China)

Terumasa Aoki, Tohoku University (Japan)

Xing-Peng Mao, Harbin Institute of Technology (China)

Xinyu Du, China Academy of Railroad Sciences (China)

Mingtao Li, National Space Science Center, CAS (China)

Jiangming Kan, Beijing Forestry University (China)

Hongping Zhou, Hefei University of Technology (China)

Guoliang Lu, Shandong University (China)

Linlin Shen, Shenzhen University (China)

Weihai Li, University of Science and Technology of China (China)

Lihua Yue, University of Science and Technology of China (China)

Yun Zhang, Kunming University of Science and Technology (China)

Wenhui Lang, Hefei University of Technology (China)

Yuesheng Zhu, Peking University (China)

Bin Yan, National Digital Switching Center (China)

Lisheng Wang, Shanghai Jiao Tong University (China)

Zhitao Xiao, Tianjin Polytechnic University (China)

Yun Tie, Zhengzhou University (China)

Tianyang Wang, Southern Illinois University, Carbondale (United States)

Zhengrui Qin, Northwest Missouri State University (United States)

Rencan Nie, Yunnan University (China)

Yuanyu Wang, Taiyuan University of Technology (China)

Bing Xiao, Shaanxi Normal University (China)

K. Ravindra, Malla Reddy Institute of Technology and Science (India)

Yan Qiang, Taiyuan University of Technology (China)

Anusha Achuthan, Universiti Sains Malaysia (Malaysia)

Jiangping Hu, University of Electronic Science and Technology of China (China)

Umair Ali Khan, Fraunhofer Institute for Integrated Circuits (Germany)

Guoyuan Liang, Shenzhen Institute of Advanced Technology (China)

Wei-Ping Zheng, South China Normal University (China)

Bingwei He, Fuzhou University (China)

Guang Yang, Beihang University (China)

Zhaoxia Xie, Beijing Institute of Graphic Communication (China) 
Ying Liu, Xi'an University of Posts and Telecommunications (China)

Meichun Yan, Hohai University (China)

Hengjian Li, University of Jinan (China)

Hongzhi Wu, Shandong Institute for Development Strategy of Science and Technology (China)

Ying Wang, Qingdao University (China)

Yang Jia, Xi'an University of Posts and Telecommunications (China)

Junjun Xiao, Harbin Institute of Technology (China)

Zhuozheng Wang, Beijing University of Technology (China)

Yin Long Wang, The Fifth Department of OEC (China)

Wei Xiong, Hubei University of Technology (China)

Jungang Han, Xi'an University of Posts and Telecommunications (China)

Shruti Bhargava Choubey, Sreenidhi Institute of Science and Technology (India)

Shi Jun, Shanghai JianQiao University (China)

Souvik Pal, Elitte College of Engineering (India)

Zhaodong Niu, National University of Defense Technology (China)

Netra Lokhande, MIT College of Engineering Kothrud (India)

Wenxing Bao, North Minzu University (China)

Yongfeng Qi, Northwest Normal University (China)

Yigang Wang, Hangzhou Dianzi University (China)

Yufeng Nie, Northwestern Polytechnical University (China)

Ying Wen, East China Normal University (China)

Xiuli Ma, Shanghai University (China)

Qiaosong Chen, Chongqing University of Posts and Telecommunications (China)

Zhiwei He, Hangzhou Dianzi University (China)

Bai Lin, Chang'an University (China)

Jingjing Si, Yanshan University (China)

Yu Zhang, Hangzhou Dianzi University (China)

Weidong Pan, University of Chinese Academy of Sciences (China)

Zhong-Gui Sun, Liaocheng University (China)

Zuochang Ye, Tsinghua University (China)

Yong Tan, Yangtze Normal University (China)

Xiaofeng Zhao, Xi' an High Tech Institute (China)

Wei Cai, Xi' an High Tech Institute (China)

Li Zhan Li, Xi' an University of Science and Technology (China)

Congli Li, Army Academy of Artillery and Air Defense (China)

Jiwei Hu, Wuhan University of Technology (China)

Peiquan Jin, University of Science and Technology of China (China)

Hao Liu, Southern Medical University (China)

Yohei Fukumizu, Ritsumeikan University (Japan)

Yun Li, Yangzhou University (China)

Yun-Hao Yuan, Yangzhou University (China)

Zhemin Zhuang, Shantou University (China)

Cheng Han, Changchun University of Science and Technology (China)

Weihai Li, University of Science and Technology of China (China) 
Xiaobo Lu, Southeast University (China)

Aiwen Jiang, Jiangxi Normal University (China)

Weiji He, Nanjing University of Science and Technology (China)

Wen Liu, Wuhan University of Technology (China)

Hongzhi Wu, Shandong Institute for Development Strategy of Science and Technology (China)

Bo Qiang, TaiYuan Satellite Launch Center (China)

Zhangjin Huang, University of Science and Technology of China (China)

Qing Zhu, Beijing University of Technology (China)

András Horváth, Peter Pazmany Catholic University (Hungary)

Feifei Tang, Chongqing Jiaotong University (China)

Zhu Li, Hangzhou Dianzi University (China)

Bin Li, University of Science and Technology of China (China)

Fengying Xie, Beihang University (China)

Jiande Sun, Shandong Normal University (China)

Songjiang Lou, Zhejiang University of Science and Technology (China)

Guoyou Wang, Huazhong University of Science and Technology (China)

Songtao Liu, Dalian Naval Academy (China)

Qingsheng Liu, Institute of Geographic Science and Natural Resources Research (China)

Volodymyr Hnatushenko, Oles Gonchar Dnipro National University (Ukraine)

Dmitriy Mozgovoy, Oles Gonchar Dnipro National University (Ukraine)

Ling Ma, Zhengzhou University (China)

Youdong Ding, Shanghai University (China)

Guoye Chen, Akita Prefectural University (Japan)

Guanzhen Yu, Longhua Hospital Affiliated to Shanghai University of Traditional Chinese Medicine (China)

Guodong Zhang, Shenyang Aerospace University (China)

Kyeong-Seop Kim, Kon-kuk University (Korea, Republic of)

Ruijun Zhang, Wuhan University of Science and Technology (China)

Yong Wang, Harbin Institute of Technology (China)

Vladislav Sergeyev, Samara National Research University (Russian Federation)

Fan Zhang, Anhui Xinhua University (China)

Houde Dai, Quanzhou Institute of Equipment Manufacturing, Haixi Institutes, CAS (China)

Zhenzhou Yu, Jilin University (China)

Jun Cai, Anhui University of Science and Technology (China)

Hung-Min Sun, National Tsing Hua University (Taiwan, China)

Xuefeng Lv, National Disaster Reduction Center of China (China)

Xiaoying Wang, Qinghai University (China)

Zheng Han, Chifeng University (China)

Zhen Ye, Chang'an University (China)

Tao Sun, Harvard Medical School, Massachussetts General Hospital, (United States)

Dmitry Kravchenko, Ben-Gurion University of the Negev (Israel) 
Yuesheng Zhu, Peking University (China)

Shaoshuai Gao, University of Chinese Academy of Sciences (China)

Wen-Ze Shao, Nanjing University of Posts and Telecommunications

(China)

Qian Huang, Hohai University (China)

Wenjun Lu, Anhui Sanlian University (China)

Jiamei Deng, Wuhan University of Technology (China)

Li-Chen Ou, National Taiwan University of Science and Technology

(Taiwan)

Su-Kit Tang, Macao Polytechnic Institute (Macao)

Rita Tse, Macao Polytechnic Institute (Macao)

Jiancheng Zou, North China University of Technology (China)

Binjie Qin, Shanghai Jiao Tong University (China)

Khurram Khurshid, Institute of Space Technology (Pakistan)

Guozhang Zhao, Capital Normal University (China)

Guohui Tian, Shandong University (China)

Shouhong Wan, University of Science and Technology of China (China)

Peiquan Jin, University of Science and Technology of China (China)

Yaowen Lv, Changchun University of Science and Technology (China)

Hui Zhao, XIOPM of CAS (China)

Ningyu Zhang, Shandong Jianzhu University (China)

Karel Horak, Brno University of Technology (Czechia)

Yong Tian, Shenzhen University (China)

Jindong Tian, Shenzhen University (China)

Jian Wang, Sichuan Agricultural University (China)

Zhi Li, Space Engineering University (China)

Bingxin Liu, Dailian Maritime University (China)

Lijun Chen, Guangzhou University (China)

Yu Zhang, Hangzhou Dianzi University (China)

Guowu Yuan, Yun Nan University (China)

Wenming Yang, Tsinghua University (China)

Langming Zhou, Hunan University (China)

Jianning Chi, Northeastern University (China)

Yi Zheng, Shandong Technology and Business University (China)

Junchao Wang, Hangzhou Dianzi University (China) 
Proc. of SPIE Vol. 11179 1117901-26

Downloaded From: https://www.spiedigitallibrary.org/conference-proceedings-of-spie on 26 Apr 2023 Terms of Use: https://www.spiedigitallibrary.org/terms-of-use 


\section{Introduction}

We had the great honor of organizing the Eleventh International Conference on Digital Image Processing (ICDIP 2019). It was truly a great pleasure for us to greet more than 180 participants from many different countries. We firmly believe that ICDIP will become an important international event in the field of Digital Image Processing.

The Eleventh International Conference on Digital Image Processing (ICDIP 2019) was hosted by Sun Yat-Sen University (China), co-sponsored by East China Normal University (China) and the International Association of Computer Science and Information Technology.

The objective of this conference was to provide a platform for the participants to report and exchange innovative ideas, up-to-date progress and developments, and discuss novel approaches to application in the digital image processing field. It is sincerely hoped that the research and development in digital image processing will be improved, and the international collaboration with common interest sharing will be enhanced.

On behalf of other co-chairs, and the organization committee of ICDIP 2019, we would like to express our heartfelt thanks to our sponsors and cooperating organizers for all they have done. Thanks also go to all the authors for their contributions to the proceedings, to all of the participants and friends for their interest and efforts in helping us to make it possible, to the program technical committee for their effective work and valuable advice, especially the conference secretary, and to the staff at SPIE for their tireless efforts and outstanding service in preparing and publishing the proceedings.

\section{Jenq-Neng Hwang Xudong Jiang}


Proc. of SPIE Vol. 11179 1117901-28

Downloaded From: https://www.spiedigitallibrary.org/conference-proceedings-of-spie on 26 Apr 2023 Terms of Use: https://www.spiedigitallibrary.org/terms-of-use 